\title{
Application of Fuzzy Similarity to Prediction of Epileptic Seizures Using EEG Signals
}

\author{
Xiaoli Li and Xin Yao \\ The Centre of Excellence for Research in Computational Intelligence and Applications, \\ School of Computer Science, The University of Birmingham, Edgbaston, \\ Birmingham, B15 2TT, UK \\ $\{x .1 i, x . y a o\}$ @cs.bham.ac.uk
}

\begin{abstract}
The prediction of epileptic seizures is a very attractive issue for all patients suffering from epilepsy in EEG (electroencephalograph) signals. It can assist to develop an intervention system to control / prevent upcoming seizures and change the current treatment method of epilepsy. This paper describes a new method based on wavelet transform and fuzzy similarity measurement to predict the seizures by using EEG signals. One part of the method is to calculate the energy and entropy of EEG data at the different scale; another part of this method is to calculate the similarity between the features set of the reference segment and the test segment using fuzzy measure. The test results of real rats show this method detect temporal dynamic changes prior to a seizure in real time.
\end{abstract}

\section{Introduction}

There are millions of people with epilepsy throughout world according to an estimate of the World Health Organization [1]. Unfortunately, at least $20 \%$ of all epileptic patients still keep seizure - free due to the inefficacy of pharmacological treatment. Therefore, many researchers attempted to develop a new method to prevent the epileptic seizures, such as chronic vagal nerve stimulation (VNS) [2] and brief burst of pulse stimulation $[3,4]$. An alternative method is to design an intervention system to inject a drug, or have a drug released once the seizure is coming [5]. The application of these methods basically dependents on the prediction of the seizures, in particular for the intervention system of a drug. Furthermore, the prediction of seizures enables patients to take as little medicine as possible, who only take it under necessary conditions rather than constantly. A warning system of the seizure prediction also may enable patients take a drug in advance to prevent from the upcoming seizures or leave some of places where is dangerous for him/her as soon as possible such as a swimming pool, a business street. The role of seizure prediction is summarized in Fig. 1

So far, a number of characterizing measures derived from nonlinear signal processing are capable of extracting information from EEG signals to predict the seizures [6-7]. Often, this non-linear method encounters two parameters (delay time and embedding dimension) determination and computation cost problems [8-10]; so they are not always applied to real EEG data, especially when the noise contribution is significant. An intelligent system has been proposed to predict the seizures [11], which is 
based on a finding that wavelet - derived energy increase before electrical and clinical seizure onset. Although intelligent techniques can learn to distinguish the pre-seizure state from the normal states, unfortunately the results from the learning methods cannot be explained in more details. From an application point of view, developing a simple and reliable prediction method is still necessary.

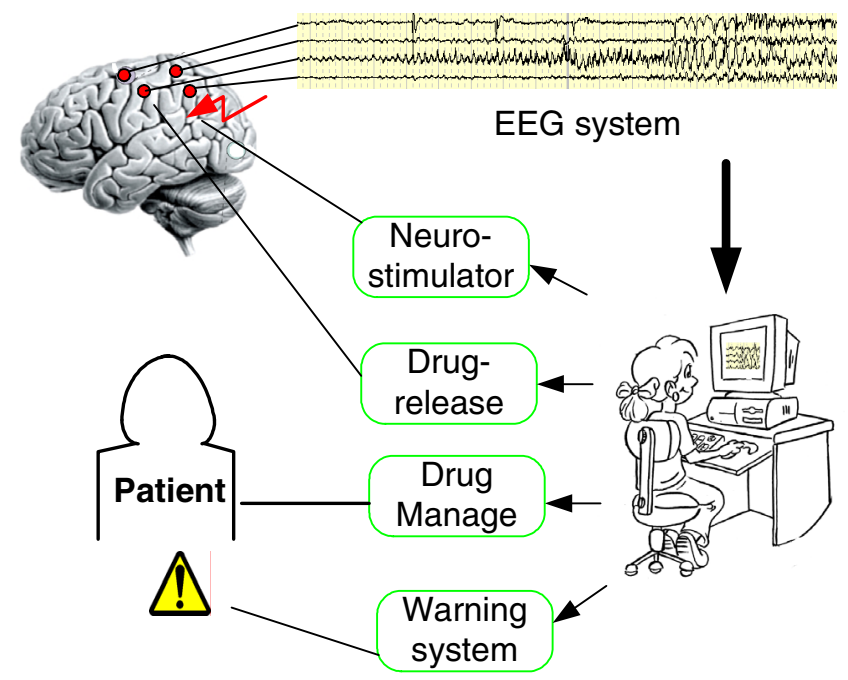

Fig.1. Block diagram of application of seizure prediction. The application of these systems is based on the seizure prediction. For a neuro-stimulator, the seizure prediction may reduce the strength of stimulation. For other three functions, they need as long prediction time as possible.

This paper proposes a new method by combining wavelet transform and fuzzy similarity measurement to predict the epileptic seizures in EEG. The first step of this method is to obtain the features from wavelet space of an EEG data, including energy and entropy of wavelet coefficients in different frequency bands. Second step is to calculate fuzzy similarity index of feature sets between the reference segment and the test segment, which integrates the energy and entropy of field potentials in different frequency bands. Finally, long-term EEG recordings of real rats are applied to test this novel prediction method. The results indicate the prediction of the epileptic seizures based on the wavelet - fuzzy similarity index could be applied in an intervention system.

\section{Method}

Often, there are five broad spectral bands of EEG signal from the clinical interest: delta $(0.5-4 \mathrm{~Hz})$, theta $(4-8 \mathrm{~Hz})$, alpha $(8-12 \mathrm{~Hz})$, beta $(12-30 \mathrm{~Hz})$, and gamma waves (30-above $\mathrm{Hz}$ ). Above five frequency bands can be extracted by using a discrete wavelet transform. The discrete wavelet decomposition of an EEG signal $x(t)$ is written as follows: 


$$
x(t)=\sum_{k=-\infty}^{+\infty} C_{J}(k) \phi\left(2^{-J} t-k\right)+\sum_{j=1}^{J} \sum_{k=-\infty}^{+\infty} d_{j}(k) \psi\left(2^{-J} t-k\right)
$$

where $d_{j}(k)$ are called wavelet coefficients at the level $j$, which can be calculated by a fast recursive scheme [12]. In this study, we only consider the energy and entropy of wavelet coefficients at each level $(j=1, \ldots, 5)$ to describe the brain state:

(1) Energy features: the energy at each resolutions level $j=1, \ldots, 5$ based on the wavelet coefficients for an segments with a sliding window ( $K$ is the length of wavelet coefficients at each resolution level $j$ ) with index $i$ is written as:

$$
\left(B E_{j}\right)^{i}=\left(\sum_{k=1}^{K} d_{j}^{2}(k)\right)^{i}
$$

(2) Entropy features: the entropy at each resolutions level $j=1, \ldots, 5$ based on the wavelet coefficients for an segments with a sliding window ( $K$ is the length of wavelet coefficients at each resolution level $j$ ) with index $i$ is given by

$$
\left(H_{j}\right)^{i}=\left(-\sum_{k=1}^{K} p_{k, j} \ln \left(p_{k, j}\right)\right)^{i}
$$

where $p_{k, j}$ is the probability density of wavelet coefficients at each resolution level $j=1, \ldots, 5$, which can be obtained by using a histogram method. Based on our experiences in [8], a biorthogonal wavelet is chosen in this study.

Then, a standard Gaussian membership function is used to describe five energy features $\left(B E_{j}\right)$ and five entropy features $\left(H_{j}\right)$ calculated $(j=1,2, \ldots, 5)$, as shown in Fig. 2. The symmetric Gaussian function depends on two parameter $\sigma$ and $c$ as given by $f(x ; \sigma, c)=\exp \left(-(x-c)^{2} /\left(2 \sigma^{2}\right)\right)$. The parameters $\sigma$ and $c$ are determined by mean and deviation of each feature $B E_{j}$ or $H_{j}$. Six membership functions is applied to describe the fuzzy character of each feature. Applying the fuzziness process for each feature, a fuzzy sets $A$ can be obtained from the energy and entropy feature sets.

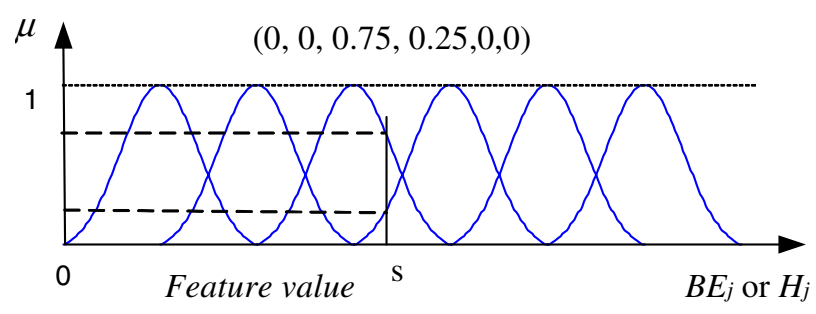

Fig. 2. Fuzzy membership functions of the features $B E_{j}$ or $H_{j}$. The horizontal axis is the feature value; on the other hand the vertical axis represent the fuzzy degree that ranges from 0 to 1 . The feature value $\mathrm{s}$ in this figure can be represented as a fuzzy set of $(0,0,0.75,0.25,0,0)$.

Suppose two fuzzy sets $A$ (present state) and $B$ (reference/normal state), and each set contains $N$ features $x_{1}, x_{2}, \ldots, x_{N}$, a simple and reliable method proposed by $[13,14]$ can be used to calculate the similarity between the two fuzzy sets, A and B, the formula is 


$$
S(A, B)=\frac{\sum_{i=1}^{N}\left(1-\left|\mu_{A}\left(x_{i}\right)-\mu_{B}\left(x_{i}\right)\right|\right)}{N}
$$

where, $1-\left|\mu_{A}\left(x_{i}\right)-\mu_{B}\left(x_{i}\right)\right|$ is regarded as the similarity degree of fuzzy set $A$ and $B$ on the features, $x_{i}$. The $S(A, B)$ is the average of the similarity degree of fuzzy $\operatorname{set} A$ and $B$, called fuzzy similarity index. The range of $S(A, B)$ is from 0 to 1 , which corresponds with the different similarity degree. $S(A, B)=1$ means the two fuzzy sets is identical, otherwise there exist a difference between the two fuzzy sets.

Main purpose of this study is to design an automated algorithm to predict the seizures by means of the fuzzy similarity index $S$. In order to obtain a baseline for the preictal state, firstly the mean value $M$ and standard deviation $S D$ of $S_{t}(t=1,2 \ldots, M)$ are calculated, which are the fuzzy similarity indexes of all interictal recordings of a subject. A local drop can be characterized by two independent parameters: its depth and duration, for any given baselines. The depth of a drop can be estimated in units of the standard deviation of the baseline epoch, whereas its duration can be quantified by the time during which the mean value of a profile drops below a certain threshold. In this study, a backward moving-average filter of width $w$ is employed to smooth the time profiles of $S_{t}$ and declared a preictal state if the smoothed profiles $S_{t}^{w}$ dropped below the interictal mean $M$ by more than $k$ standard deviations $S D$ :

$$
P S=\left\{\begin{array}{l}
1 \text { if } S_{t}^{w}<M-k^{*} S D \\
0 \text { otherwise }
\end{array}\right.
$$

where $P S=1$ stands for a preictal state. Two parameters $k$ and $w$ govern the mean depth of a drop over a certain time. In order to determine suitable values for the two parameters, the sensitivity and specificity rate of preictal state detection should be considered. The depth parameter $k$ varied from 0 to 6 interictal standard deviations while the duration parameter $\mathrm{w}$ varied from 0 to $10 \mathrm{~min}$ in this study.

All of procedure of the method is summarized in Fig. 3. The procedure contains a discrete wavelet transform, feature extraction, fuzziness, reference state setting, threshold settings, fuzzy similarity index calculation and indication of the preictal state.

\section{EEG Recordings}

In this paper, an epilepsy model with bicuculline is applied. 14 Sprague-/Dawley rats are tested. Prior to surgery, the rats are anesthetized with an i.p. injection of Nembutal (sodium pentobarbital, $65 \mathrm{mg} / \mathrm{kg}$ body weight). One electrode is placed on the skull with dental acrylic. During the experiments, every precaution is taken to minimize suffering to the animals. Rat is initially anesthetized with pentobarbital $(60 \mathrm{mg} / \mathrm{kg}$, i.p.) while body temperature is maintained $\left(36.5-37.5^{\circ} \mathrm{C}\right)$ with a piece of blanket. The degree of anesthesia is assessed by continuously monitoring the EEG, and additional doses of anesthetic are administered at the slightest change toward an awake pattern (i.e., an increase in the frequency and reduction in the amplitude of the EEG waves). Then, bicuculline i.p. injection is used to induce the rat epileptic seizures. EEG sig- 
nals are recorded using an amplifier with band-pass filter setting 0.5-100 Hz. Seizure onset times are determined by visual identification of a clear EEG seizure discharge. The detail of the recording of EEG data and the determination of epileptic onset time can be found in [9-10].

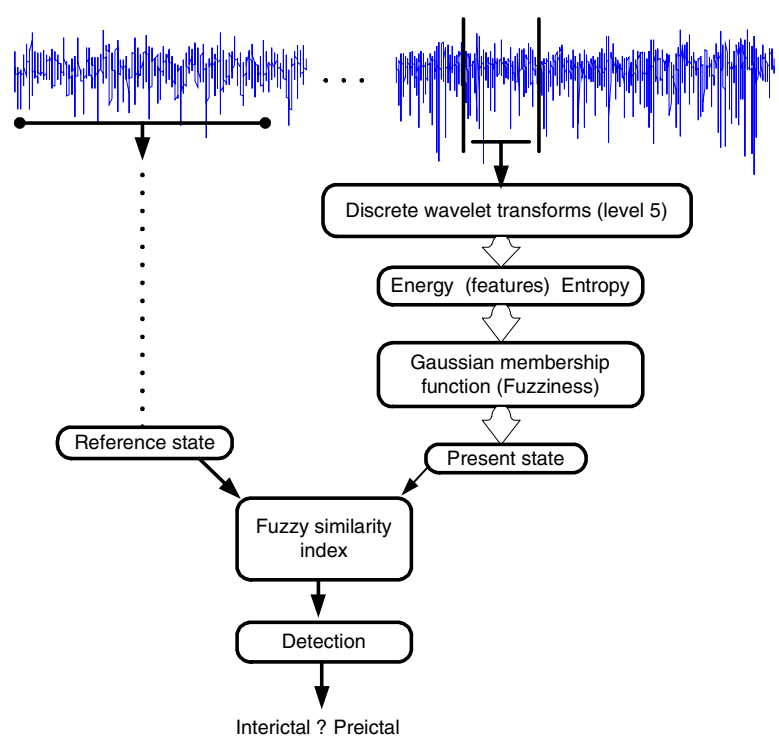

Fig. 3. Illustration of complete procedure of the prediction method for the epileptic seizures. The method is composed of two parts. (Left) one is to build the reference states from the interictal, which is so far from ictal. Another is to calculate the features of current state and the fuzzy similarity index with a reference state, then to identify the current state whether or not is a preictal state by using the detection method.

\section{Results}

The EEG recording of rats are analyzed using a moving - window technique, which are divided into segments of 1000 sampling points each, corresponding to a window length of $5 \mathrm{~s}$ at the given sampling rate $(200 \mathrm{~Hz})$, and the window overlapped by $50 \%$ so the distance in time between the starting points of two consecutive windows is 2.5 s. Prior to the calculation of the fuzzy similarity, two steps of data processing are carried out for each data window: (1) discrete wavelet decomposition; and (2) calculation of energy and entropy of wavelet coefficients. The prediction method is carried out for all 14 animal tests. We only show one typical example in this paper.

Fig. 4 shows the application result of the proposed method to long-term EEG recordings covering 20 minutes. At 6:41(minute:second), the bicuculline is injected to induce the epileptic seizures. The beginning of epileptic seizure occurs at 
12:27(minute:second). The first three minutes EEG recordings are treated as a reference state. Hopefully the reference segment should include the most of salient features of the interictal EEG signal. Then, the fuzzy similarity index with the reference state is obtained by moving window over the entire EEG recordings, as shown in Fig. 4 (bottom) after smoothed. The three vertical lines are the injection time, the preictal start time and the seizure start time from left to right, the horizontal long line is threshold, where the $\mathrm{k}$ and $\mathrm{d}$ are selected as 3.5 and 2 according to the testing of some EEG signals. The plot reveals that the fuzzy similarity index gradually decreases during the preictal period. The preictal state is detected when the fuzzy similarity index is less than the threshold. In this test, the beginning point of preictal state is at 10:15(minute:second), therefore the preictal duration is 102 seconds. The quantification of 20 min recordings can be carried out in less than $30 \mathrm{~s}$ using Matlab on a P6 2 $\mathrm{G}$ personal computer.

Fig. 5 summarizes the seizure anticipation time of 14 animals. The results confirm that the method proposed can predict the seizures in most of the animals (13/14) several minutes (seconds) in advance (mean $109.6 \mathrm{~s}$ ). It is seen that the various anticipation times for the 14 animals are highly variable in the duration. These results suggest that seizure emergence is a complex, non-repetitive process even for similar animals.
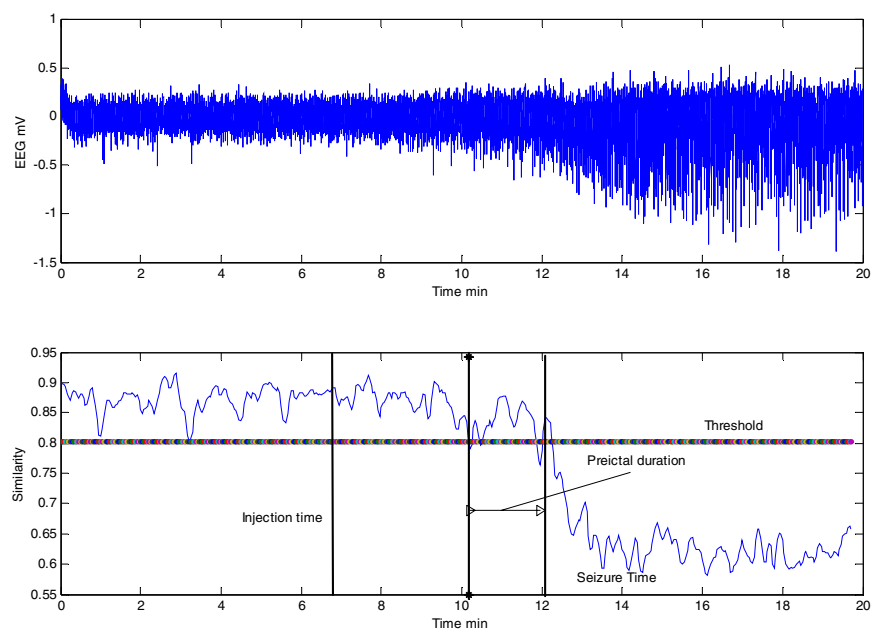

Fig. 4. Top: EEG recordings; Bottom: Preictal state detection. At $6: 41$, the bicuculline is injected. The epileptic seizure occurs at 12:07. The beginning point of the preictal state is at 10:15. The preictal duration is about 1:52.

\section{Conclusions}

A prediction method of epileptic seizure should meet following requirements [5]: (a) the method is able to run real-time robustly and on-line in a clinical setting with 


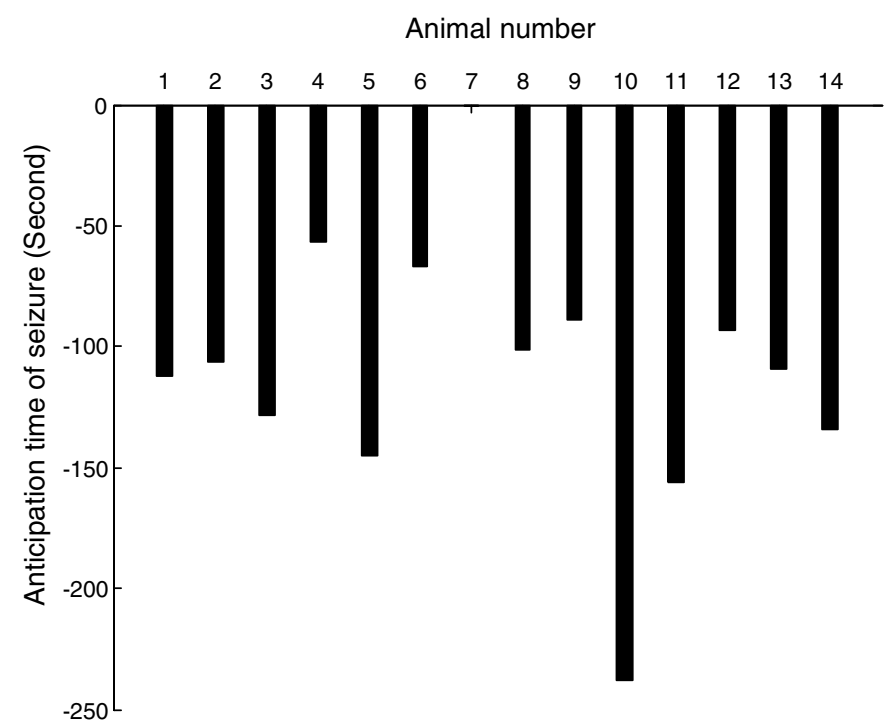

Fig. 5. The anticipation time of 14 animals by using the fuzzy similarity index. The mean time of the seizure anticipation is $109.6 \mathrm{~s}$. The method gave a false prediction of seizure for animal 7.

noised EEG; (b) it possesses very good sensitivity and specificity in long-term continuous recordings across animals or patients; (c) it can provide a long-term warning before each seizure and an accurate prediction of the occurrence time of the impending seizure at the time of the warning.

The advantages of the method in this paper are summarized. (i) Features extractions with wavelet transform. Wavelet transform are very meaningful because the hidden information of EEG can be revealed; the noise effort can also be reduced because some data under some scales is omitted. (ii) Significant advantage of this method is able to check directly the fuzzy similarity index between two windows, rather than differences between quantities evaluated separately. Thus, the threshold for the detection of preictal is not very hard to determine by the fuzzy similarity index of each objective, this method is not influenced by the biological variability. (iii) The computational cost of the new method relatively low and can be used in real time for clinical application. (iv) The method does not finally require specific prior knowledge for each objective. Therefore, this novel method could be applied a practical intervention system.

\section{Acknowledgements}

We are grateful to the support of Wellcome Trust and AMD UK. We also thank John Jefferys for his helpful comments. 


\section{References}

1. Litt, B., Echauz, J.: Prediction of epileptic seizures. The Lancet Neurology. 1(2002) 22-30

2. Fisher, R. S., Krauss, G. L., Ramsay, E., Laxer, K., Gates, J.: Assessment of vagus nerve stimulation for epilepsy: report of the therapeutics and technology assessment subcommittee of the American Academy of Neurology. Neurology. 49(1997) 293-297

3. Lesser, R. P., Kim, S. H., Beyderman, L.: Brief bursts of pulse stimulation terminate afterdischarges caused by cortical stimulation. Neurology. 53(1999) 2073-2081

4. Motamedi, G. K., Lesser, R. P., Miglioretti, D. L.: Optimizing parameters for terminating cortical afterdischarges with pulse stimulation. Epilepsia. 43(2002) 836-846.

5. Iasemidis, L.D.: Epileptic seizure prediction and control. IEEE Transactions on Biomedical Engineering. 50(2003) 549-558

6. Lehnertz, K.: Non-linear time series analysis of intracranial EEG recordings in patients with epilepsy - an overview. International Journal of Psychophysiology. 34(1999) 45-52

7. Winterhalder, M., Maiwald, T., Voss, H.U., Aschenbrenner-Scheibe, R., Timmer, J., Schulze-Bonhage, A.: The seizure prediction characteristic: A general framework to assess and compare seizure prediction methods. Epilepsy and Behavior. 4(2003) 318-325

8. Li, X., Guan, X., Du, R.: Using damping time for epileptic seizures detection in EEG. Modelling and Control in Biomedical Systems (Edited by David Dagan Feng and Ewart Carson), Elsevier Ltd, (2003) 255-258

9. Li, X., Kapiris, P.G., Polygiannakis, J., Eftaxias, K.A., Yao, X.: Fractal spectral analysis of pre-epileptic seizures phase: in terms of criticality. Journal of Neural Engineering, 2 (2005) 11-16

10. Li, X. Ouyang, G., Yao, X., Guan, X.: Dynamical Characteristics of Pre-epileptic Seizures in Rats with Recurrence Quantification Analysis, Physics Letters A. 333 (2004) 164-171

11. Geva, A.B., Kerem, D.H.: Forecasting generalized epileptic seizures from the EEG signal by wavelet analysis and dynamic unsupervised fuzzy clustering. IEEE Transactions on Biomedical Engineering. 45(1998) 1205-1216

12. Meyer, Y.: Wavelets, Applications and Algorithms, Siam, 1993

13. Hyung, L. K., Song, Y. S., Lee, K. M.: Similarity measure between fuzzy sets and between elements. Fuzzy Sets and Systems. 62(1994) 291-293

14. Wang, W. J.: New similarity measures on fuzzy sets and on elements. Fuzzy Sets and Systems. 85 (1997) 305-309 